\title{
Anesthetic Management of Septic Shock
}

\author{
Konstantinos Alexopoulos (Meds 2016) \\ Faculty Reviewer: Dr Ramiro Arellano, MD, FRCPC (Department of Anesthesiology)
}

\section{BACKGROUND}

Severe sepsis, a systemic inflammatory response characterized by acute organ dysfunction in the face of an infectious process, is a major healthcare issue worldwide. Sepsis presents a unique challenge in the management of patients who require anesthesia and definitive surgical treatment. Anesthesiologists are involved in the multidisciplinary management of patients with severe sepsis throughout the patient's clinical course. ${ }^{1}$ The mainstays of management include prompt administration of IV antibiotics in conjunction with preoperative fluid and pharmacological resuscitation. The goal is to optimize end organ perfusion through the judicious use of pressors, inotropes and IV fluids. ${ }^{2}$ An example might include a patient with an acute small bowel obstruction complicated by sepsis. The patient would be appropriately resuscitated using a multi-modal approach as outlined above, while definitive management would include surgical decompression of the obstruction. Intra-operatively, these patients require conscientious induction of anesthesia, ample fluid resuscitation coupled with invasive hemodynamic/biochemical monitoring. ${ }^{1}$ Efforts to promote a favourable surgical outcome of a septic patient begins before the surgeon picks up the scalpel.

\section{CASE PRESENTATION}

Patient SM is a 67-year-old retired female who presented to University Hospital with nausea, vomiting, constipation and acute kidney injury. SM's creatinine trended upwards for several days from 341 to 372 then 401 . Kidney ultrasound was performed which revealed severe bilateral hydronephrosis. Subsequently, SM had bilateral ureteric stents to relieve the outlet obstruction. Despite the ureteric stents, SM's urine output remained poor and an IV fluid bolus was administered. Within 24 hours, SM developed increasing $\mathrm{SOB}$ and $\mathrm{O}_{2}$ requirement, as well as a cough with fever. SM was started on IV Ceftriaxone and Piperacillin-Tazobactam to cover gram negative organisms that colonize the urogenital tract including E. coli, Proteus and Klebsiella. ${ }^{3}$ Interventional radiology was consulted for the placement of bilateral nephrostomy tubes to relieve the bilateral ureteric outlet obstructions.

SM was evaluated by the consultant anesthesiologist, resident physician and author KA. SM's past medical history included GERD, chronic fatty liver, hypothyroidism, irritable bowel syndrome, dyslipidemia, psoriasis, migraines and anxiety. SM appeared much older than her stated age with central morbid obesity and a BMI of $48 \mathrm{~kg} / \mathrm{m}^{2}$. With this information SM was assigned the American Society of Anesthesiologists number 5 physical status classification. Class 5 patients are not expected to survive 24 hours without surgical intervention.4 SM's vitals at this time were: NIBP $95 / 42 \mathrm{mmHg}$, MAP calculated at $60 \mathrm{mmHg}, \mathrm{HR}$ of $130 \mathrm{bpm}, \mathrm{SpO}_{2}$ of $88 \%$ on $10 \mathrm{~L}$ nasal cannula, febrile with a temperature of $39.2^{\circ} \mathrm{C}$. Neurologically the patient was intact, alert and oriented to person, place and time. However, as the exam continued SM became increasingly obtunded, showing signs of laboured breathing. Her airway exam revealed a Mallampati Class IV with decreased thyromental distance. Intubation was predicted to be extremely difficult. SM's most recent blood work and chemistries showed a leukocytosis with a developing coagulopathy due to urosepsis. As the patient was a Jehovah's Witness, all blood products were refused. The patient had a $20 \mathrm{Ga}$ IV in situ. Plan for induction of anesthesia included an awake arterial line for accurate hemodynamic monitoring, and an additional large bore IV (> 18 Ga), modified rapid sequence induction and oral endotracheal intubation using the Glidescope and transfer to the Medical Surgical Intensive Care Unit (MSICU) for postoperative care.

An arterial line was established in the right radial artery using sterile technique. A good waveform was observed. The dicrotic notch indicates closure of the aortic valve and the wash back of blood against the aortic valve. The waveform showed a lower than normal dicrotic notch indicating that the patient was severely hypovolemic. ${ }^{5} \mathrm{SM}$ was having difficulty cooperating with our instructions as she was extremely somnolent. Tracheal topicalization was achieved with $2 \%$ lidocaine spray. BP had dropped to $85 / 40 \mathrm{mmHg}$. A 1 litre fluid bolus of $0.9 \% \mathrm{NaCl}$ was administered prior to intubation. Hypnotic agents like etomidate were discussed for the maintenance of what little hemodynamic stability SM had, but ultimately was not chosen. Evidence in the literature indicated that etomidate in sepsis can precipitate marked adrenal insufficiency and increase rates of mortality. ${ }^{6}$ The patient's potassium was $3.8 \mathrm{mmol} / \mathrm{L}$ which made succinylcholine our choice for neuromuscular blockade. Remifentanil, a potent ultra-short-acting opioid was chosen to assist visualization of the glottis during intubation. Remifentanil in rat models has demonstrated a protective effect against sepsis. The proposed mechanism is a decreased release of inflammatory mediators IL-1, IL-6, TNF-alpha, while also decreased the inducible nitric oxide synthase which is a potent endogenous vasodilator. ${ }^{7}$

A defasciculating dose of Rocuronium $3 \mathrm{mg}$ IV was administered to prevent fasciculations and myalgias from succinylcholine. Midazolam $1 \mathrm{mg}$ was administered for anxiolysis. Cricoid pressure was applied to the larynx. IV Lidocaine was not administered since it was deemed that the pharyngeal response of increased BP to laryngoscopy would be favourable in this patient. Remifentanil 30 mcg was administered, followed by Propofol $30 \mathrm{mg}$ and Succinylcholine $100 \mathrm{mg}$. At this point, $\mathrm{SM}^{\mathrm{S}} \mathrm{SpO}_{2}$ was $85 \%$ despite administration of $100 \% \mathrm{O}_{2}$. The Glidescope was used and a McCormack-Lehane Grade II view was obtained. Intubation was successful and confirmed with end tidal $\mathrm{CO}_{2}\left(\mathrm{ETCO}_{2}\right)$ return and equal bilateral 
air entry. Patients with sepsis have a decreased anesthetic requirement, thus, maintenance of anesthesia was achieved using Sevoflurane at a concentration of $1 \%$ end-tidal Sevoflurane, which is one half its minimal alveolar concentration. ${ }^{2} \mathrm{SM}^{\prime} \mathrm{ETCO}_{2}$ was 21 $\mathrm{mmHg}$, indicating poor perfusion likely due to decreased cardiac output and developing pulmonary edema secondary to acute respiratory distress syndrome (ARDS). Phenylephrine $200 \mathrm{mcg}$, a pure alpha-1 agonist was administered, with little to no effect. Another $300 \mathrm{mcg}$ was given with no observable clinical effect.

SM was now markedly hypotensive at $70 / 35 \mathrm{mmHg}$ as per the arterial line. Treatment for pulmonary edema caused by ARDS is positive end expiratory pressure (PEEP), but PEEP decreases venous return and results in hypotension. At this point, the need for adequate ventilation did not outweigh the need for hemodynamic stability. Vasopressin was considered but ultimately was not administered in favour of more powerful direct acting inotropes. $10 \mathrm{mcg}$ of IV epinephrine was administered in conjunction with a fluid bolus. The radiologist began to scrub for the procedure. SM developed tachycardia at $145 \mathrm{bpm}$, with a $\mathrm{BP} 55 / 30 \mathrm{mmHg}$. $\mathrm{SpO}_{2}$ was $78 \%$. Sevoflurane was switched off; oxygen at $100 \%$, flows at $15 \mathrm{~L} / \mathrm{min}$. IV epinephrine was increasingly administered in $10 \mathrm{mcg}$ boluses escalating to $30 \mathrm{mcg}$ boluses. BP improved slightly to $70 / 40 \mathrm{mmHg}$. An infusion of epinephrine/norepinephrine was established.

The anesthesiologist advised the radiologist that the patient was too unstable for the procedure. SM's coagulopathy and sepsis were far too severe, and in the event of blood loss the risk for mortality was greatly increased. ${ }^{2} \mathrm{SM}$ was transferred to the MSICU on the infusion of epinephrine/norepinephrine and cardiac monitors. SM was started on continuous renal replacement therapy and maintained on an epinephrine/norepinephrine infusion. 24 hours later, the ureteric stents were changed at the bedside in the MSICU. Prior to the procedure, SM received DDAVP (Vasopressin) $20 \mathrm{mcg}$ IV and Vitamin K $10 \mathrm{mg}$ IV for her coagulopathy. The procedure was a success and the patient's coagulopathy started to resolve. SM remained in the MSICU for 4 days requiring decreasing doses of pressors. After a 24 day stay, she improved markedly and was discharged home in stable condition.

\section{DISCUSSION}

This case presented some difficult decisions from a management perspective. As always it is important to remember that physicians inadvertently can cause harm to their patient(s). From iatrogenic infections, to ill-informed decision making, in some cases, the gold standard treatment for the patient may result in deleterious consequences. In SM's case, the decision to forego the procedure in the face of marked hypoxemia and hypotension was the most appropriate management. In the event that plan A begins to fail, plan B must already be formulated and ready to implement, while remembering: First, do no harm.

\section{REFERENCES}

1. Eissa D, Carton EG, and Buggy DJ. Anaesthetic management of patients with severe sepsis. Br J Anaesth. 2010 Oct; 105 (6): 734-743.

2. Yoon SH. Concerns of the anesthesiologist: anesthetic induction in severe sepsis or septic shock patients. Korean J Anesthesiol. 2012 Jul; 63 (1): 3-10.

3. Kalra OP, Raizada A. Approach to a patient with urosepsis. J Glob Infect Dis. 2009 Jan-Jun; (1) :57-63.

4. Daabis M. American society of anaesthesiologists physical status classification. Indian J Anaesth. 2011 Mar-Apr; 55 (2): 111-115.

5. McGhee BH, Bridges EJ. Monitoring arterial blood pressure: what you may not know. Crit Care Nurse. 2002 April; 22 (2): 60-79.

6. Chan CM, Mitchell AL, Shorr AF. Etomidate is associated with mortality and adrenal insufficiency in sepsis: a meta-analysis. Crit Care Med. 2012 Nov; 40(11): 2945-53. 